\title{
Hierarchical Management Architecture for Multi-Access Networks
}

\author{
Dzmitry Kliazovich, Tiia Sutinen, Heli Kokkoniemi-Tarkkanen, Jukka Mäkelä, and Seppo Horsmanheimo \\ VTT Technical Research Centre of Finland \\ Vuorimiehentie 3, Espoo, 02044 VTT, Finland \\ Email: firstname.lastname@vtt.fi
}

\begin{abstract}
Future Internet will be highly heterogeneous supporting a multitude of access technologies with overlapping coverages. The automation and optimization of network operations like resource, mobility or QoS management in such a multi-access and multi-operator environment becomes a very challenging but vital task in order to ensure smooth network operation and user satisfaction. Cognitive network management is seen as the solution for this. However, any self* mechanism designed for small-scale networks and requiring an accurate view of the whole network status for decision-making will not be able to meet the future needs. In this paper, we propose a novel multi-access network management architecture targeted for large heterogeneous multi-access and multi-operator networks. The architecture introduces hierarchy to network management to ensure scalability. We also present results obtained from a case example of the proposed decision-making solution implemented to our cognitive network testbed.
\end{abstract}

Index Terms-Network Expert System, cognitive network management, policy-based, handovers.

\section{INTRODUCTION}

In the future, mobile communications is characterized with network access heterogeneity and concurrent utilization of multiple access networks for optimal service. Network operators are thus faced with the challenge of managing and maintaining networks of multiple technologies including, for example, LTE, HSPA, WLAN, and WiMAX. In addition, users are forced to ponder their access options to select the most appropriate one for the task at hand, which they typically see as inconvenient. To decrease the burden of multiaccess network management for the operators and to hide all the technical complexity of network selection and handovers from the users, automation through cognitive management mechanisms are seen as the solution.

According to a general definition from [1], cognitive network management involves intelligent network elements that observe the network conditions; plan, decide and act based on the obtained information; as well as learn from their earlier decisions and adapt their operation accordingly. The mechanisms are used for improving different aspects of network performance, such as resource management, Quality of Service (QoS), access control, etc., in the end-to-end scope of a data flow. For implementing the decision-making and learning, different technologies can be applied, such as SelfOrganized Map (SOM) [2], Bayesian networks [3], and fuzzy logic [4]. The decision-making is typically controlled with policies which provide means for operators and users to have control over automation.

Cross-layer communications and network monitoring play a key role in providing the required context information for cognitive network management. Several cross-layer communication frameworks have been proposed over the recent years to facilitate multi-access network management and especially to improve handover performance in heterogeneous networks. These include, for instance, the IEEE 802.21 Media Independent Handover Services framework [5] as well as the triggering framework [6]. In addition, the network monitoring tools available today, including various QoS monitoring tools (e.g. QoSMeT [7]) and network probes (e.g. [8]), are able to collect a vast amount of performance data from coexisting networks for the decision makers.

Considering a future multi-access and multi-operator network environment, it is clear that network management mechanisms designed for small-scale networks and requiring an accurate view of the whole network status in decision-making do not scale well - neither in respect of the decision-making accuracy nor the signalling overhead. A distributed and hierarchical management architecture for localized control and management is needed instead. In this paper, we propose a novel multi-access network management architecture targeted for heterogeneous multi-access and multi-operator environments. The architecture is an extension to the signalling and decision-making architectures we have presented in [8], [9]. It also has similarities to the access network selection and discovery functions of the 3GPP system architecture [10], but is not tied to any specific standard.

In the context of our proposed architecture, in this paper, we define the different levels of decision-making supported by the architecture as well as their interactions. We also present a case example of the proposed decision-making solution implemented to our cognitive network testbed. The test case illustrates the ability of a network to detect congestion in an access link and to instruct a mobile node to react accordingly.

\section{SyStem ARCHITECTURE}

Fig. 1 illustrates the proposed Hierarchical Control and Management (HCAM) architecture for mobile multi-access networks. HCAM is an extension of Distributed Control and Management Framework (DCMF) proposed by us in [9]. It 
aims at enabling real-time performance optimization and adaptive self-management of network entities and mobile terminals. It can be seen as a process that keeps track of current network conditions, analyses, plans, and makes the decisions based on these conditions. Furthermore, the process constantly controls the results of made decisions and assesses their quality. Based on such analysis, it constantly learns evolving its decisionmaking ability.

In Fig. 1, each operator owns a multi-access network while the mobile devices are assumed to be capable of roaming within the boundaries of a single access network as well as between different access technologies.

A number of network specific probes are deployed in the access networks. Network resource probes are the components capable of collecting performance information from the network nodes like access points or routers. For collecting the information e.g. from WLAN access points, probes may use Simple Network Management Protocol (SNMP). The information harvested by probes is fed into the nearest Network Expert System (NES).

\section{A. Network Expert Systems}

Hierarchical Network Expert Systems (NESs) form the core of the HCAM. Fig. 2 presents the details of HCAM operation at different levels of hierarchy. Each NES includes a learning cycle when it tries to classify or map existing trends in the monitored data, the process of data monitoring and decisionmaking events. Each NES tries to resolve performance and quality of user experience issues at its own level of hierarchy and, only when not capable, it requests an assitance from a higher-level NES. There are four types of NES systems defined according to the operation point in the network:

1) Access Network NES: serves a segment of the operator access network. Multiple probes deployed in the access network supply it with the setup and performance information harvested from the base stations and other network elements. The task of the access network NES is in resolving terminal performance problems within a single access technology and providing a horizontal handover, if needed. Vertical handovers and inter-network control are the tasks of the Operator NES described further.

The algorithms implemented in NES are technology independent. However, many of them operate on a predefined set of parameters and require a training phase. In practice, the setup parameters and performance indicators are not the same for different access technologies. Therefore, a dedication of NESs per access network is architecturally feasible and advisable. For example, even similar parameters like the level of error correction may require different treatment. In WLAN the errors are corrected with retransmissions at the link layer solely while WiMAX and 3G networks implement Hybrid Automatic Repeat reQuest (HARQ) schemes [11] as a combination of retransmissions and Forward Error Correction (FEC) techniques.

2) Operator NES: is the main decision-making entity in the operator network. It follows predefined operator policies and controls access network NESs.

During its ordinary operation each access network should be able to resolve mobile terminal performance issues within its boundaries. The actions are taken only in case a significant deviation from the required QoS values is experienced. If the performance shortage cannot be resolved by an access network NES, the network state is reported to operator NES, which attempts to find a solution at the higher level of hierarchy. To do so, it may either attempt to make a decision based on the performance information already provided by the access network NESs or to issue a control command for increasing the amount of monitoring from a given segment of the network for a more precise analysis.

In large operator networks, the operator NES receives only aggregated performance information from access network NESs describing a network segment as a whole rather than coming from individual base stations. However, in small operator networks the resolution and amount of monitoring can be increased automatically making a decision system more centralized. In a similar way, the amount of signaling can be controlled dynamically based on the network load level and the time of the day.

3) Inter-operator NES: stands at the highest level of the HCAM hierarchy, but has a limited influence at the operator network performance. Instead, it is focused at performance optimization of the connections roaming out of the operator network.

The inter-operator NES is guided by the inter-operator policies. This serves the cases when the operator network cannot provide a required quality due to the lack of communication resources, coverage, or an increased interference malfunctions. In such a situation, it becomes more advisable to hand this connection over to another operator. Even at the cost of the lost revenue, it leads to a better satisfaction level of the end user.

4) Mobile Expert System (MES): resides inside a mobile terminal. It maintains an explicit knowledge about currently running user applications, their Quality of Service (QoS) requirements, and a set of current communication performance parameters provided by the mobile terminal. To do so, MES interfaces with each protocol layer monitoring its internal configuration and operational parameters. For example, the frequency spectrum, received signal strength, and modulation details are monitored at the physical layer; the link state and the strength of error correction at the link layer; the network state, routing and mobility details at the network layer; and the available bandwidth, delay, delay jitter, and packet loss rate are accessed at the transport layer.

By monitoring communication experience and satisfaction levels of user applications, MES derives knowledge about which combination of setup parameters on which radio channels leads to improvements and forms a knowledge base. The MES's interface to the access network is used to deliver performance alerts triggered, in case application QoS demands are not satisfied and the performance drawbacks cannot be resolved at the mobile terminal locally. 


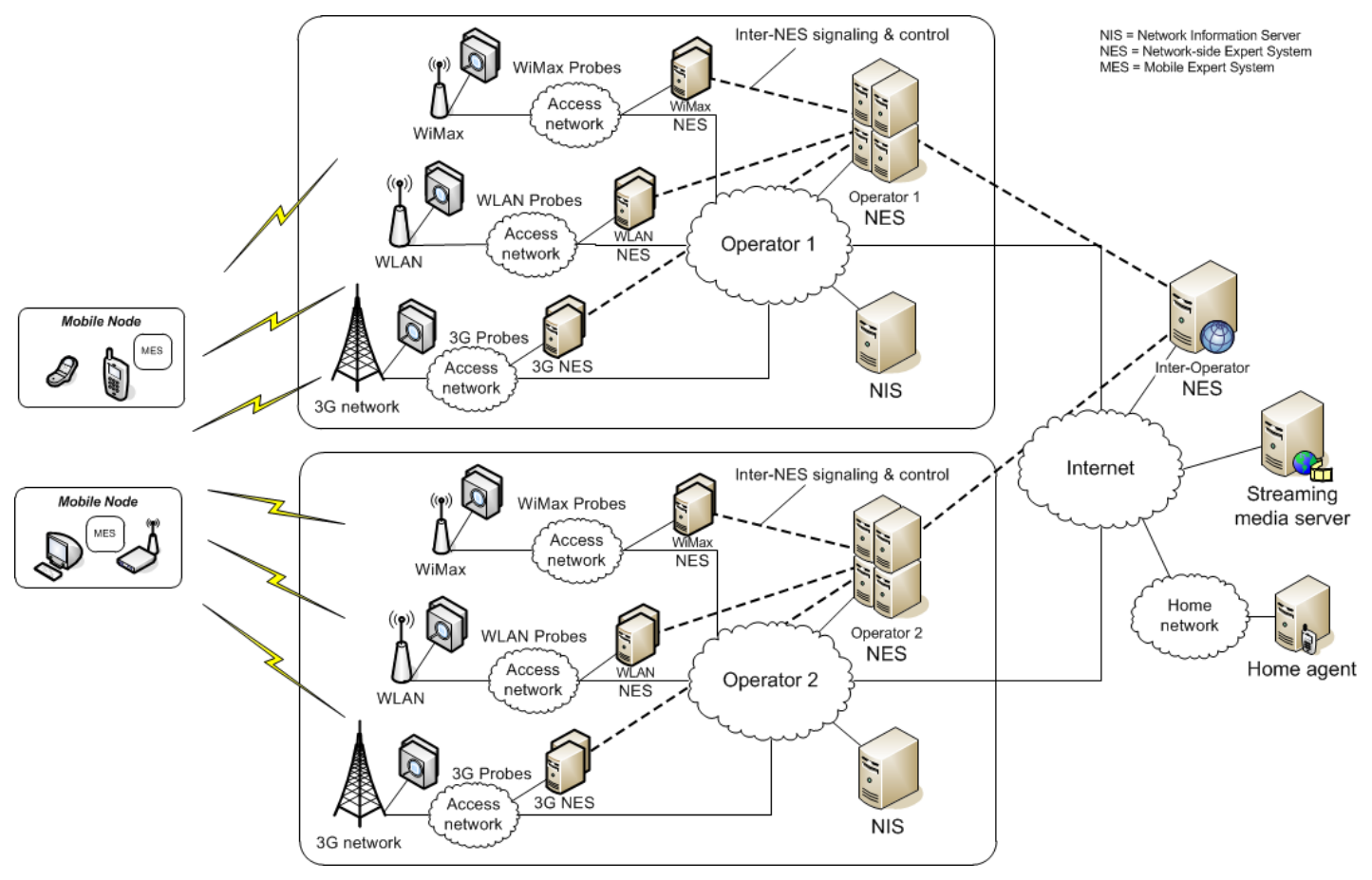

Fig. 1. Hierarchical network expert systems architecture.

Depending on the mobile terminal capabilities, the functionalities of MES are adjusted accordingly. When high-end smartphones or laptops are used and there is no shortage of computational and data storage resources the MES performs comprehensive monitoring and advanced decision-making. However, for low-end terminals it reduces decision-making functionality significantly and simply aggregates and forwards performance measurements and user satisfaction levels to the network core.

\section{B. NESs signaling}

Efficient information signaling between HCAM entities is one of the key factors determining the overall system performance. The information harvested at the base stations need to be transmitted to probes. The monitored information is aggregated to messages and sent to neighboring NESs. NES entities in its turn exchange aggregated signaling and control information. The universal signaling technique employed in HCAM is Triggering Engine (TRG) proposed by us in [6]. In contrast to the IEEE 802.21 standard [5] the TRG is not constrained by only lower protocol layers or a direction of signaling. Each network entity (NES, MES, or a probe) can register itself with TRG and send a trigger carrying an id, a type, and a value fields towards another entity. Moreover, a trigger can be originated from a hardware device, a protocol layer implemented in kernel space, or from a user-space application. As soon as the trigger is delivered, it is up to the subscriber to decide how to process and react to the received information. For more details on TRG implementation, trigger formats and processing procedures the reader is referred to [6].

\section{Operating Policies}

The HCAM operation is driven by a set of policies defined by terminal users, operators, and inter-operator relations.

1) User policies: guide MES operation implemented in mobile terminals. They are directed to maximize Quality of Experience (QoE) and smooth operation of user applications. The QoE is represented with both subjective and objective measures of the overall customer satisfaction level by the service [12].

In case of a shortage of communication resources, the user policies specify which applications may scarify their QoE. For example, delay tolerant applications may be selected to detain their requests releasing resources for more critical applications carrying voice or multimedia data. In case of adaptive multimedia, the bandwidth requirement of the service can also be decreased through rate adaptation.

User policies become extremely relevant when economic aspects of multiple access networks are concerned. They feed MES with instructions for balancing between QoE and different subscription plans. For example, cellular networks are typically more expensive than WLAN or WiMAX, but offer more stable channels with well-defined bandwidth and delay characteristics [13]. Therefore, it is advisable for a terminal to stay within WLAN or WiMAX boundaries as long as QoE remains at the desired level. However, if not, the user policies step in. It is obvious that voice (or VoIP) connections are likely to be handed over to cellular network to resolve QoE issues. For high data rate connections such as a file transfer or video streaming, a user may instead wish to leave them on WLAN or WiMAX where the data transmission costs are less expensive. User policies may also specify different QoE thresholds for 


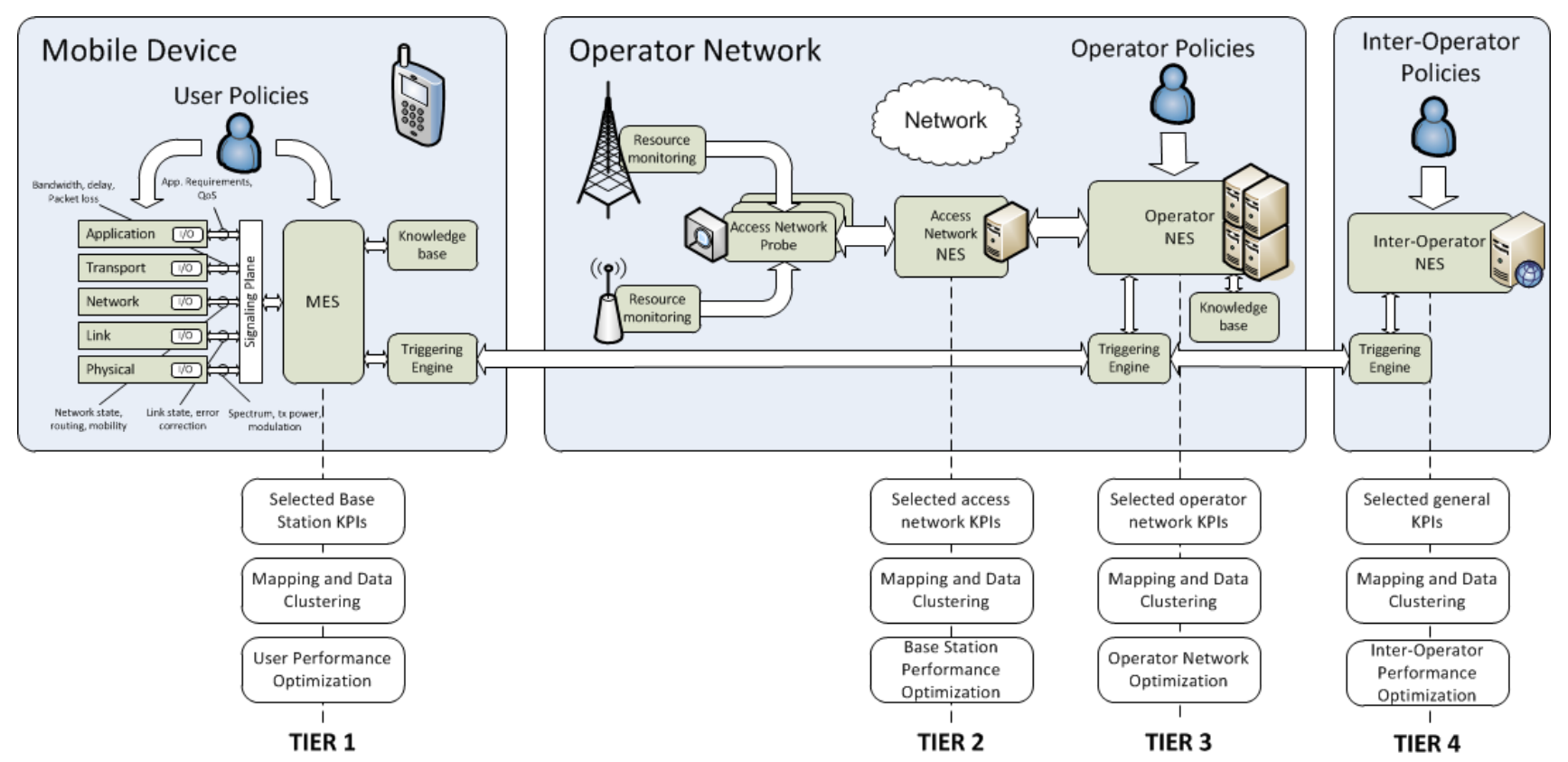

Fig. 2. HCAM framework.

different network technologies tolerating lower QoE for less expensive connections.

2) Operator policies: specify operator resource allocation, user admission, and billing policies. They drive operations of operator NES and access network NESs.

The most common trend in resource allocation is focused on load balancing of the network resources in time, frequency and spatial domains while keeping the QoS requirements satisfied [14]. However, the rules become more complicated when different types of services and different billing strategies come into play. For example, most operators treat voice connections with higher priority, since they are the main source of the revenue, prioritizing them during resource allocation over data connections. In a similar way, conventional voice connections prevail on the VoIP traffic.

Multiple access networks belonging to the same operator open a new frontier in service provisioning policies. In such situation, an operator while keeping the pricing policies unchanged may shift a portion of user traffic to a more expensive network in order to resolve performance bottlenecks in a transparent way. For example, cellular networks are often overprovisioned or underutilized especially in certain hours of the day or geographical areas. The operator may take a decision for the ordinary WiMAX subscriber to route a portion of its VoIP traffic through a cellular connection at no additional charge. This will increase the QoE for WiMAX users at no cost for the operator.

3) Inter-operator policies: reflect resource management agreements between operators. They control inter-operator NES. Being placed in the root of the NES tree the interoperator NES is responsible for handling cases when a certain subscriber request cannot be accommodated by the operator network with a requested QoS level. In such cases, it is handed over and serviced on another operator's network. Even at the cost of the lost revenue such scheme can increase a customer satisfaction level and be beneficial for the operator.

For network operators owning limited or even no infrastructure (virtual operators) the inter-operator relations form the core of their business model. In such a way, the interoperator NES is envisioned to become an essential part of the marketplace where operators place their bids for network resources in a highly dynamic manner.

Fig. 3 presents a message exchange sequence between different NES entities in the case of inter-operator handover. Whenever it is not possible to resolve performance issues within a single network, the access network NES issues corresponding notification to the operator NES (message 2). The operator NES tries to consider handing over the questioned connections to the different access technology network. However, if not possible the inter-operator NES should be contacted. To facilitate the process, the mobile terminal is requested to provide the list of networks it senses. Having such information inter-operator NES is able to suggest the best roaming option considering quality characteristics and congestion levels as well as inter-operator agreements and available billing options. Finally the handover notification is delivered to MES at the mobile terminal.

Having no precise information on individual base stations and radio channels the inter-operator NES can only make generic suggestions at the network segment level. Then, it is up to the MES to make the concrete base station selection. It may use passive scanning or active probing techniques to determine the best base station. After inter-operator roaming further adjustment of QoS parameters may be performed by the nearest access network NES. 


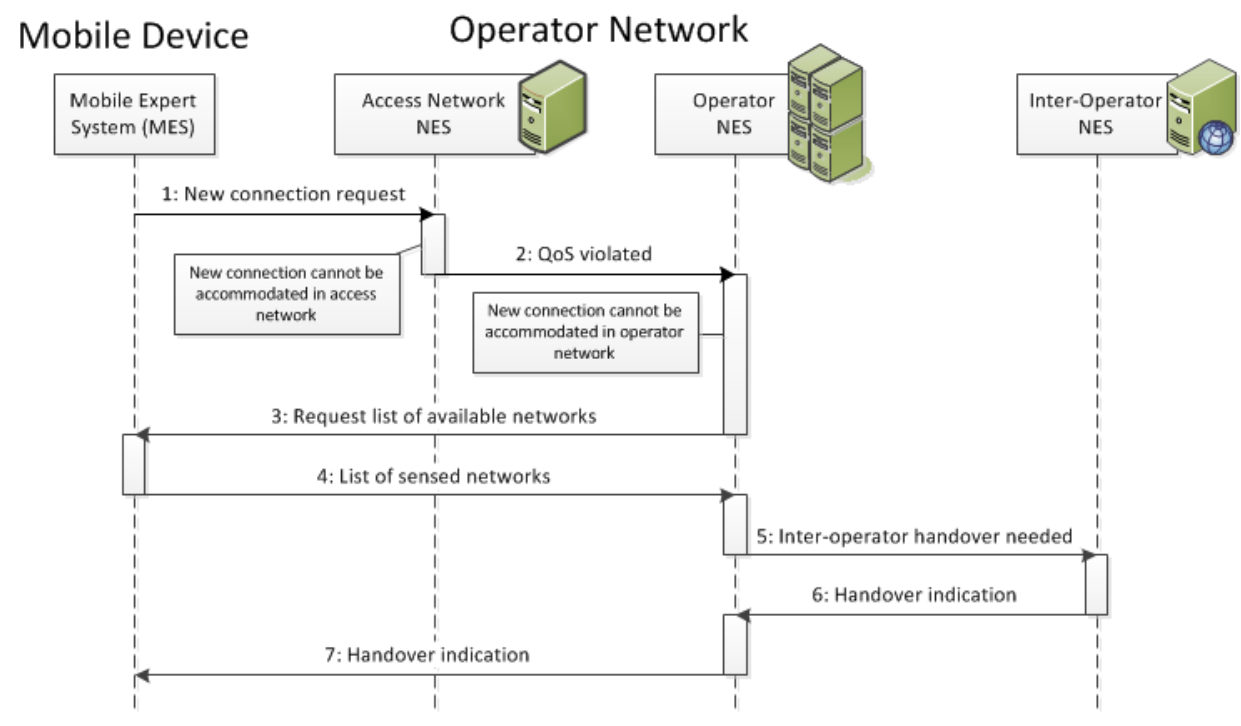

Fig. 3. Message flow during inter-operator handover.

\section{Experimental Evaluation: A Test CASE}

A special testbed has been developed to evaluate the performance of the proposed HCAM architecture concept. It is composed of two WLAN Access Points (APs) providing the connectivity to up to five client laptop the number of which depends on the test case. A single WLAN probe monitors both APs and delivers the obtained measurement to the NES component. A set of 20-30 of the most relevant parameters is selected for monitoring. Furthermore, the WLAN probe is also capable of generating Key Performance Indicators (KPIs) used for NES training. For more details on the testbed setup the reader is referred to [8].

There are two types of traffic sources available in the network: traffic pattern generators and streaming video servers. During the NES training phase, a constant bit rate generator was used. It increases its transmission rate in a stair-step fashion for up to the AP congestion level. A snapshot of the training data is illustrated in Fig. 4. Here, the load level of one client node increased from 2 to $14 \mathrm{Mb} / \mathrm{s}$ in $1 \mathrm{Mb} / \mathrm{s}$ steps while other clients received $1 \mathrm{Mb} / \mathrm{s}$ streams constantly. A UDP protocol is selected at the transport level in both directions to provide a stable bit rate and avoid possible disturbances from the TCP flow control and error correction mechanisms. Each stair-step is approximately one minute long.

While the upper part of Fig. 4 presents a training sequence the bottom part (yellow line) outlines the evolution of one of the key performance indicators generated by NES, called radio interface quality. It can be observed that the increased traffic load and growing congestion at the radio link turns the radio interface quality from high to low at some point.

After the NES is trained, it can operate real-time. For every event received, it compares it to its knowledge base and, if needed, generates triggers to perform adaptation. Each decision may contain several actions requested.

Fig. 5 presents NES operation results in the scenario with

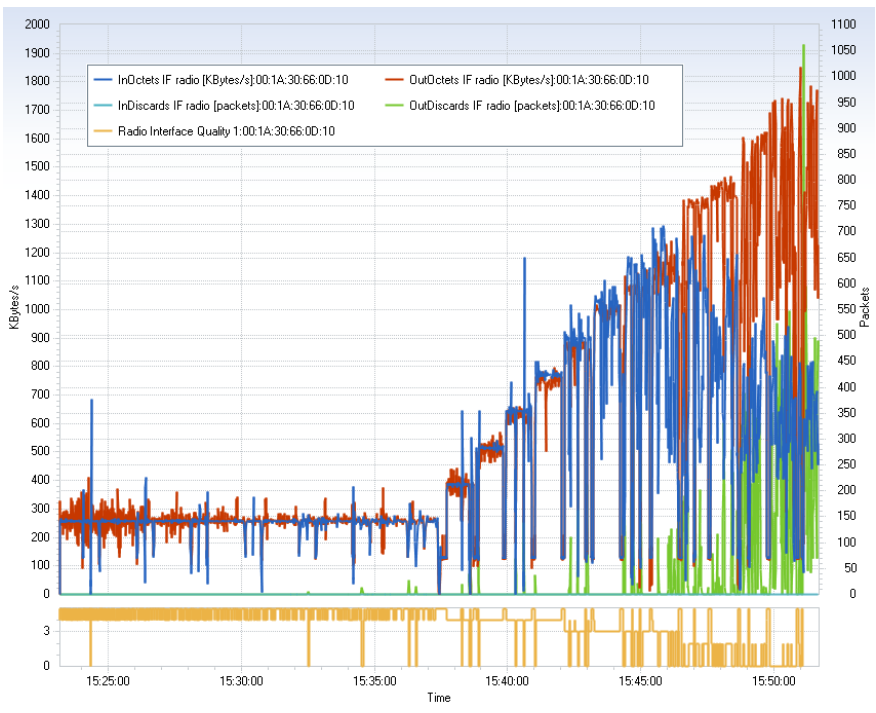

Fig. 4. An example of training data.

network congestion. A set of action rules and corresponding triggers has been defined. One of the rules is specified to issue triggers like Inform about AP's status, Inform status of both networks together, or Handover recommended whenever congestion occurs or the evidence of congestion is detected. In this figure, the first step illustrates a situation when the AP is about to get congested, but the mobile client is not aware of it, because the control traffic is not affected while the signal strength and quality indicators correspond to a good channel. The second step illustrates a more severe congestion which is also experienced at the mobile client.

The obtained results confirm NESs ability to detect growing network congestion long before any packets start to be discarded for the overflown buffers. The blue line at the bottom of Fig. 5 shows triggers sent by NES during this test run. For 


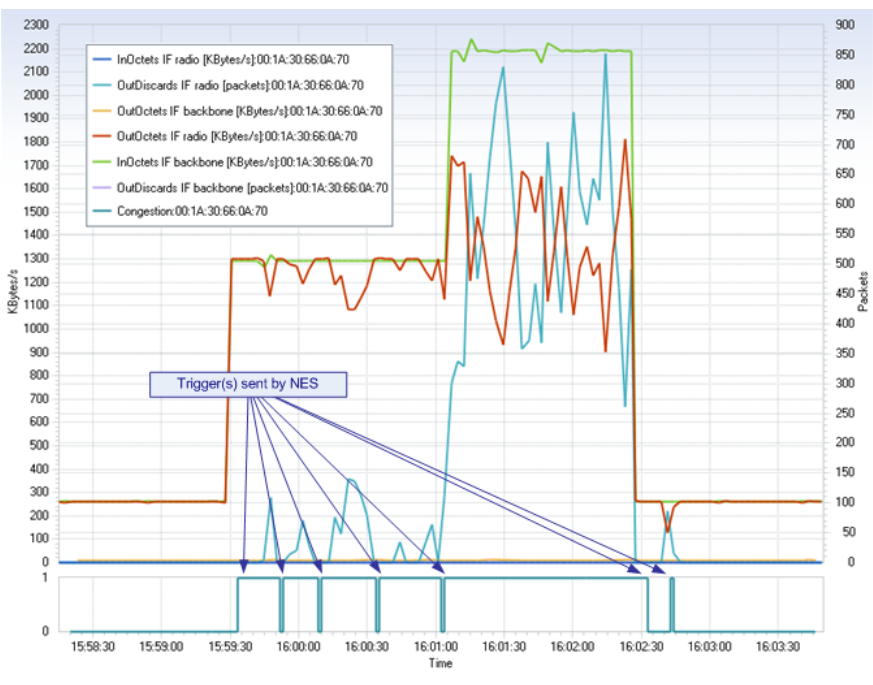

Fig. 5. Results of the test run.

every change from 0 to 1 a trigger indicating congestion is sent to the mobile node while for every change from 1 to 0 a trigger indicating end of congestion is released. If the congestion is not severe, some of the data samples may indicate that the congestion is over and NES sends unnecessary triggers. Therefore, the reaction time to the changes must be carefully considered. A sudden response can easily create a Ping-Pong effect.
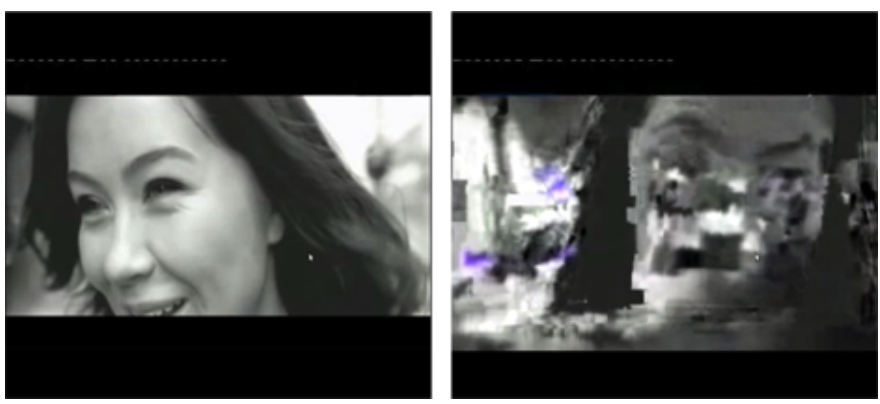

Fig. 6. Users video experience during the handover with and without the congestion monitoring

To better illustrate the advantages for mobile nodes and end user, we run the test with Mobile IPv6 [15] handovers between two WLAN access points with real time video streaming. Test showed that with the help of NES and HCAM the packet delay remains constant and only 90 packets were lost during the handover. This amount of packet loss didn't have major effect to the user QoE and only a small glitch in the video could be seen. Without having any monitoring cababilities MIP does not make the handover at all due to the fact that MIP binding update messages managed to be transported through the congested network and no congestion could be recognized on the mobile node side. Fig. 6 shows the MIP handover case with and without the the help of HCAM and NES.

\section{ACKNOWLEDGEMENTS}

The authors would like to acknowledge the funding support from the EU FP7 in the framework of UNIVERSELF project and VTT Technical Research Centre of Finland in the framework of the Intelligent Telecommunication Systems with Enhanced Cognitive Processing (ITSE) project as well as the research fellowship provided by the European Research Consortium for Informatics and Mathematics (ERCIM).

\section{Conclusions}

This paper proposes a novel control and management architecture HCAM for large multi-access and multi-operator networks. The decision making is performed by a number of network expert systems arranged into a tree. The HCAM operation is driven by a set of policies defined by terminal users, operators, and inter-operator relations.

The results obtained from the testbed experiments confirm HCAM's ability to detect and resolve performance bottlenecks to provide an increased level of QoE for the majority of user applications. The HCAM architecture allows network operators to optimize configuration and network management in highly dynamic network environments where manual control is often not feasible. A proper distributed control and management architecture is envisioned as an enabling component for building future multi-access multi-operator networks.

\section{REFERENCES}

[1] R. Thomas, L. DaSilva, and A. MacKenzie, "Cognitive networks," in DySPAN 2005, Baltimore, MD, USA, Nov. 2005, pp. 352-360.

[2] J. Vesanto and E. Alhoniemi, "Clustering of the self-organizing map," IEEE Transactions on neural networks, vol. 11, no. 3, 2000.

[3] F. V. Jensen and T. D. Nielsen, Bayesian Networks and Decision Graphs. Second Edition Springer Verlag, 2007.

[4] Fuzzy Cognitive Maps: Advances in Theories, Methodologies, Applications and Tools. Springer, 2010.

[5] Media Independent Handover Services, IEEE Std. 802.21, 2009.

[6] J. Mäkelä and K. Pentikousis, "Trigger management mechanisms," in ISWPC'07, San Juan, Puerto Rico, Feb. 2007.

[7] J. Prokkola, M. Hanski, M. Jurvansuu, and M. Immonen, "Measuring WCDMA and HSDPA delay characteristics with QoSMeT," in ICC'07, Glasgow, Scotland, Jun. 2007.

[8] S. Horsmanheimo, J. Eskelinen, and H. Kokkoniemi-Tarkkanen, "NES network expert system for heterogeneous networks," in ICT2010, Doha Qatar, Apr. 2010.

[9] J. Mäkelä, M. Luoto, T. Sutinen, and K. Pentikousis, "Distributed information service architecture for overlapping multiaccess networks," Multimedia Tools and Applications, pp. 1-18, 2010.

[10] J. Sachs and M. Olsson, "Access network discovery and selection in the evolved 3GPP multi-access system architecture," Eur. Trans. Telecomms., 2010.

[11] R. Comroe and J. Costello, D., "Arq schemes for data transmission in mobile radio systems," Selected Areas in Communications, IEEE Journal on, vol. 2, no. 4, pp. 472 - 481, Jul. 1984.

[12] P. Brooks and B. Hestnes, "User measures of quality of experience: why being objective and quantitative is important," Network, IEEE, vol. 24 , no. 2 , pp. $8-13,2010$.

[13] C. Krapichler, "Lte, hspa and mobile wimax a comparison of technical performance," in Hot Topics Forum: LTE vs WiMAX and Next Generation Internet, 2007 Institution of Engineering and Technology, 2007, pp. $1-31$.

[14] W. Song, W. Zhuang, and Y. Cheng, "Load balancing for cellular/wlan integrated networks," Network, IEEE, vol. 21, no. 1, pp. 27 -33, 2007.

[15] D. Johnson, C. Perkins, and J. Arkko, "Mobility Support in IPv6," ser. Request for Comments, no. 3775. IETF, Jun. 2004. 